Please do not remove this page

RMIT

UNIVERSITY

\title{
4-dimensional trajectory generation algorithms for RPAS mission management systems
}

Gardi, Alessandro Giacomo Maria; Ramasamy, Subramanian; Sabatini, Roberto

https://researchrepository.rmit.edu.au/esploro/outputs/9921862846701341/filesAndLinks?institution=61 RMIT_INST\&index=null

Gardi, A. G. M., Ramasamy, S., \& Sabatini, R. (2015). 4-dimensional trajectory generation algorithms for RPAS mission management systems. Proceedings of the IEEE International Conference on Unmanned Aircraft Systems (ICUAS 2015), 386-394. https://doi.org/10.1109/ICUAS.2015.7152314

Document Version: Accepted Manuscript

Published Version: https://doi.org/10.1109/ICUAS.2015.7152314

Repository homepage: https://researchrepository.rmit.edu.au

(C) 2015 IEEE

Downloaded On 2023/04/26 21:31:07 +1000

Please do not remove this page 
Thank you for downloading this document from the RMIT Research Repository.

The RMIT Research Repository is an open access database showcasing the research outputs of RMIT University researchers.

RMIT Research Repository: http://researchbank.rmit.edu.au/

\section{Citation:}

Gardi, A, Ramasamy, S and Sabatini, R 2015, '4-dimensional trajectory generation algorithms for RPAS mission management systems', in Proceedings of the IEEE International Conference on Unmanned Aircraft Systems (ICUAS 2015), United States, 9-12 June 2015, pp. 386-394.

See this record in the RMIT Research Repository at:

https://researchbank.rmit.edu.au/view/rmit:33436

Version: Accepted Manuscript

Copyright Statement: (C) 2015 IEEE

Link to Published Version:

http://dx.doi.org/10.1109/ICUAS.2015.7152314 


\title{
4-Dimensional Trajectory Generation Algorithms for RPAS Mission Management Systems
}

\author{
Alessandro Gardi, Subramanian Ramasamy and Roberto Sabatini \\ School of Aerospace, Mechanical and Manufacturing Engineering \\ RMIT University, Melbourne, VIC 3000, Australia \\ roberto.sabatini@rmit.edu.au
}

\begin{abstract}
This paper presents the algorithms enabling real-time 4-Dimensional Flight Trajectory (4DT) functionalities in Next Generation Mission Management Systems (NG-MMS), which are the core element of future Remotely Piloted Aircraft Systems (RPAS) avionics. In particular, the algorithms are employed for multiobjective optimisation of 4DT intents in various operational scenarios spanning from online strategic to tactical and emergency tasks. The adopted formulation of the multi-objective 4DT optimisation problem includes a number of environmental objectives and operational constraints. In particular, this paper describes the algorithm for planning of 4DT based on a multi-objective optimisation approach and the generalised expression of the cost function adopted for penalties associated with specific airspace volumes, accounting for weather, condensation trails and noise models.
\end{abstract}

Keywords-Trajectory Optimization; 4-Dimensional Trajectory; Flight Management System; Air Traffic Management.

\section{INTRODUCTION}

In order to progress towards a more sustainable aviation sector, it is of paramount importance to increase environmental and economic performances, evolving the design, operation and auxiliary tasks associated with aircraft operations (e.g., support and disposal), with a holistic approach. While the evolutions in the design of an aircraft, of its components and of its reference mission can introduce significant benefits, an essential consideration has to be given to its operation in real air traffic scenarios. An integrated approach to Communication, Navigation, Surveillance/Air Traffic Management (CNS/ATM) and Avionics (CNS+A) is the essential link to ultimately enact the various improvements offered by new technologies and concepts in the operational context. It is envisaged that the adoption of 4-Dimensional Trajectory (4DT) concepts in conjunction with an increased use of automation and airto-ground data-link communications in an Intent-Based Operations (IBO) context will provide the most significant benefits. In this perspective, ongoing research has proposed the introduction of novel ground-based CNS/ATM systems that, in conjunction with airborne avionics, will enable IBO [1-3]. In line with this research path, we propose the introduction of a groundbased 4DT Planning, Negotiation and Validation (4-PNV) system that, together with airborne Next Generation Mission Management Systems (NG-MMS) enables optimised 4DT intent generation, negotiation and validation in real-time [4-7]. The optimisation of 4DT intents is one of the most promising and growing applications in the CNS+A context. Historically, flight planning was performed in a sort of pattern search between the available air routes; subsequently, an optimal cruise level was calculated, and finally the vertical profiles for climb and descent were determined. As a matter of fact, although the optimisation of flight trajectories has been extensively studied in the last decades, substantial potential benefits are left unattained due to the limited addressing of the operational specificities and due to the limited modelling and availability of the dynamic real-time information updates.

\section{CNS+A CONCEPT OF OPERATIONS}

The 4-PNV system allows 4DT planning, data exchange and negotiation/validation with the NG-MMS. The optimised 4DT intents generated by the NG-MMS are downlinked to the ground-based 4-PNV system via a Next Generation Aeronautical Data-Link (NG-ADL). The 4-PNV system receives multiple 4DT intents from each aircraft, allowing for various options to identify a conflict free solution for each aircraft, in real-time [8]. Providing multiple trajectory options decreases the length of negotiation time and reduces the need for ATM systems to calculate optimal trajectories remotely. Once all optimal conflict-free trajectories have been identified, reviewed and approved by the human operators, the 4DT is finally validated, as illustrated in Fig. 1.

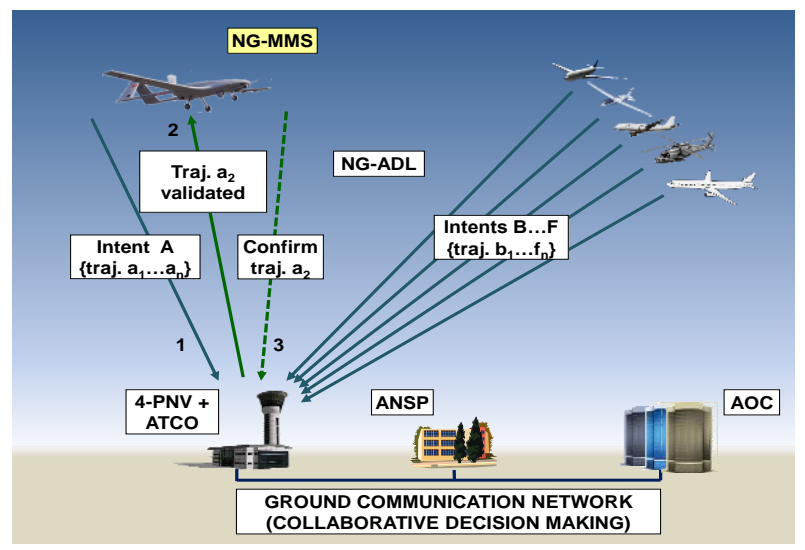

Fig. 1. CNS+A concept of operations. 
When feasible trajectories cannot be identified among the NG-MMS intents, the 4-PNV acknowledges the NG-MMS and, based on the performance weightings collaboratively agreed between the Airline Operating Centres (AOC) and the Air Navigation Service Provider (ANSP), calculates a new family of optimal trajectories (AOC/ANSP intent) and uplinks them to the aircraft. The NG-MMS then analyses the received intent, assessing the compliance with aircraft performance and operational envelope, and sends a confirmation to the ground. The increased automation allows more aircraft to fly optimal flight paths, limiting the intervention of the controllers to higher-level and emergency decisions. Increased airspace flexibility will enable greener and more efficient flight profiles. The Collaborative Decision Making (CDM) is substantially enhanced through the exploitation of automated negotiation schemes.

\section{NG-MMS}

Conventionally, MMS act as key enablers of automated navigation and guidance services in RPAS.
The MMS function is to reduce the ground control pilot's workload by acting both as a mission planner and a mission monitor. Additionally, due to the growing concern for environmental sustainable solutions, significant research is currently carried out to improve operational efficiency and safety, while reducing the impacts of aviation on environment. In this context, the NG-MMS are key enablers for generating globally optimal trajectories that fulfill the evolving operational, safety and environmental requirements. The NG-MMS is developed for 4DT-IBO in combination with the 4PNV systems and NG-ADL. The efficiency and effectiveness of 4DT planning, negotiation and validation functionalities of the NG-MMS are directly driven by the nature of information sharing. The NGMMS modules can be modified for adaptive, autonomous, aided and deliberate operations as listed in Table I. The NG-MMS thus addresses all elements of the 10-level scale of autonomy, which are based on the decision-maker (human or system) and on how the decisions are executed.

TABLE I. ClasSIFICATION OF AUTONOMY LEVELS (ADAPTED FROM [9]).

\begin{tabular}{|c|c|c|c|}
\hline Operations & Control & Sheridan Scale & Human-Systems Interaction Approach \\
\hline Adaptive & Executive & $9-10$ & $\begin{array}{c}\text { System performs all aspects of decision-making and informs operator } \\
\text { after the execution, if required, per preplanned criteria or operator } \\
\text { request. }\end{array}$ \\
\hline Autonomous & Supervisory & $6-8$ & $\begin{array}{c}\text { System generates decision alternatives and a preferred option for } \\
\text { execution and informs operator in time for intervention. }\end{array}$ \\
\hline Aided & Consent Based & $3-5$ & $\begin{array}{c}\text { System generates decision alternatives and recommends one to carry } \\
\text { out - but only with operator approval. Operator may select alternative } \\
\text { option. }\end{array}$ \\
\hline Deliberate & Manual & $1-2$ & $\begin{array}{c}\text { System executes commands initiated by operator (system may } \\
\text { provide and/or recommend decision alternatives to operator). }\end{array}$ \\
\hline
\end{tabular}

These levels are based on information acquisition, information analysis, decision and action selection, as well as action implementation functions. The NG-MMS architecture is primarily based on the core functionalities namely flight planning (FPLN), localisation and state determination, trajectory optimisation (TRAJ), performance predictions (PRED) and guidance. Additionally, the MMS also provides auto-throttle controls for engines and communicates with novel ground-based 4-PNV systems. The NG-MMS software is based on multi-objective and multi-model 4DT optimisation algorithms. The primary NG-MMS modules are:

1. Trajectory Planning/ Optimisation - This module performs 4DT planning and optimisation functions for pre-tactical, tactical and emergency situations. The 4DT optimiser includes the models pool and constraints pool involves a number of cost functions. A number of cost functions are introduced for optimisation including minimisation of fuel consumption, flight time, operative cost, noise impact, emissions and contrails. The databases include navigation, performance, magnetic deviation and environmental databases. The 4DT planner and optimiser blocks are illustrated in Fig. 2. The implementation of 4DT optimisation algorithms as part of the identified system architecture modules allows for the development of TBO aspects. The NG-MMS also includes cooperative and noncooperative DAA function software modules.

2. Trajectory Monitoring - It performs state estimation, calculating the deviations between the active 4DT intents and the estimated/predicted aircraft states.

3. Path Correction - It corrects the path deviation in terms of lateral, vertical and time profiles and the generated steering commands are provided to the guidance module of the NG-MMS.

4. Trajectory Negotiation and Validation - It carries out the process of negotiation that can be initiated 
by the pilot via the NG-MMS, making use of the information available on board, or by the NG-ATM system, supervised by the Air Traffic Control Operator (ATCO).

5. MMS Performance Manager - It monitors the active 4DT intents for errors to address integrity requirements. The integrity analysis module is based on RNP, RCP and RSP managers.

6. MMS Integrity Manager - This module is used to generate integrity caution (predictive) and warning (reactive) flags based on inputs from different sensors/systems and predefined decision logics. A loss of data leads to re-initialising of the trajectory planning and subsequently the 4DT generation and optimisation process. For instance, the main causes of GNSS signal outage and degradation in flight, namely: antenna obscuration, multipath, fading due to adverse geometry and Doppler shift are identified and modelled to implement integrity thresholds and guidance algorithms in the Avionics-Based Integrity Augmentation (ABIA) system [19-21].

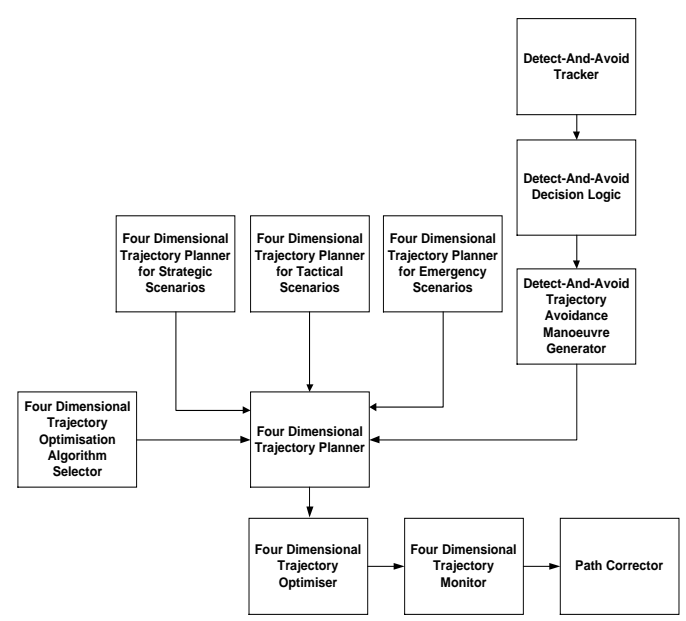

Fig. 2. NG-MMS 4DT planner and optimiser.

The negotiation and validation of 4DT intents by the NG-MMS / 4-PNV system is dependent on:

- On-board validation based on synchronization, sufficient fuel, compliance with dynamics (time performances, turn performances, speed, altitude), obstacle separation, locally sensed weather and compliance with the Integrated Vehicle Health Management (IVHM) system regarding aircraft health status and other aspects.

- Ground-based validation based on air traffic separation (lateral, vertical, longitudinal), sector occupancy, airspace restrictions (special use areas) and time based restrictions (night time noise abetment procedures).

With the increasing levels of automation in air transportation, integrity monitoring and augmentation systems have gained enormous significance. Errors affecting the CNS+A systems (e.g., pseudo-range GNSS observables) are taken into account in evaluating the CNS performances. As illustrated in Fig. 3, the integrity analysis module provides inputs to the Integrity Flag Generator (IFG) based on the errors affecting the CNS systems. The IFG uses a set of predefined Caution and Warning Integrity Flags (CIF/WIF) threshold parameters to trigger the generation of both caution and warning flags associated with CNS performance degradations. In case a warning flag is generated, a recapture command is used to trigger the 4DT regeneration and optimisation process.

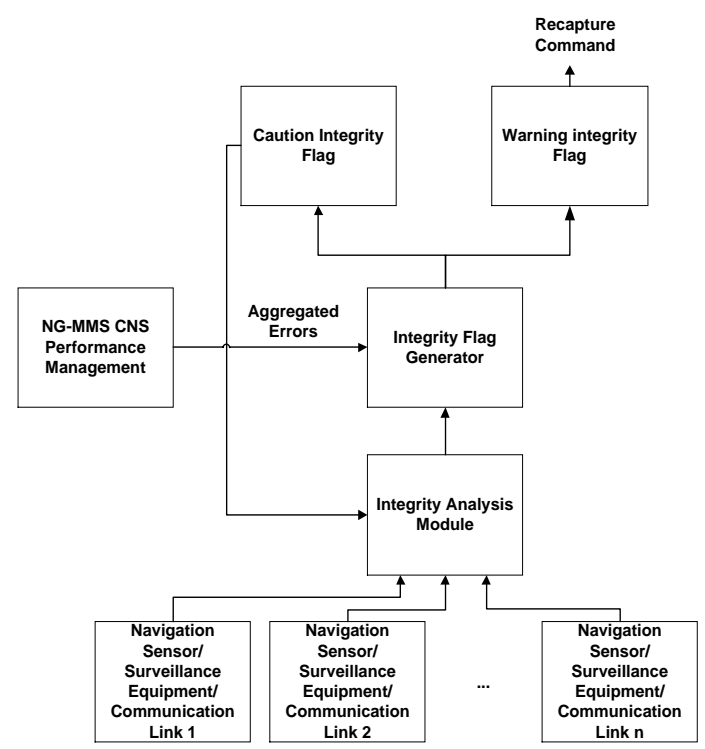

Fig. 3. NG-MMS integrity monitoring.

Fig. 4 is a schematic block diagram of the NG-MMS performance management modules. The performance management tasks are defined for all $\mathrm{CNS}+\mathrm{A}$ parameters. These modules receive data from the 4DT planner/optimiser module. The RNP, RSP and RCP integrity performance management modules provide data to the integrity management blocks that in-turn generate CIF/WIF. The $\mathrm{CNS}+\mathrm{A}$ performance management blocks are interfaced with the 4-PNV system. Figure 5 is a schematic block diagram of the $\mathrm{CNS}+\mathrm{A}$ systems for RPAS. The optimisation of 4DT trajectories is performed on-board by the NG-MMS. A number of RPAS equipped with NG-MMS are controlled by the ground command, control and intelligence system aided by LOS and BLOS communication links. Human Machine Interface and Interactions $\left(\mathrm{HMI}^{2}\right)$ at the Ground Control Station (GCS) are equipped with navigation, tactical, health management and engine management displays. The ground inter-communication system consists of a ground-to-ground communication network between the 4-PNV system, Air Navigation Service Provider (ANSP) and the Airline Operation Centre (AOC). The overall NG-MMS architecture is illustrated in Figure 6. 


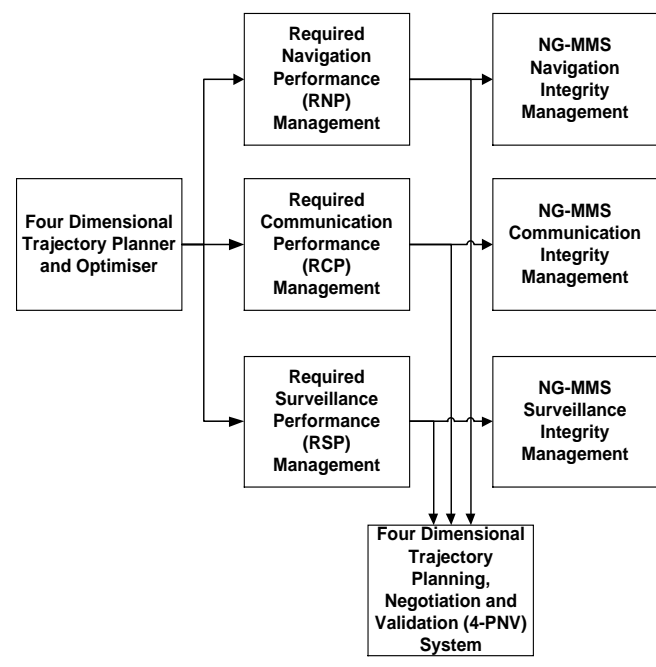

Fig. 4. NG-MMS performance management.

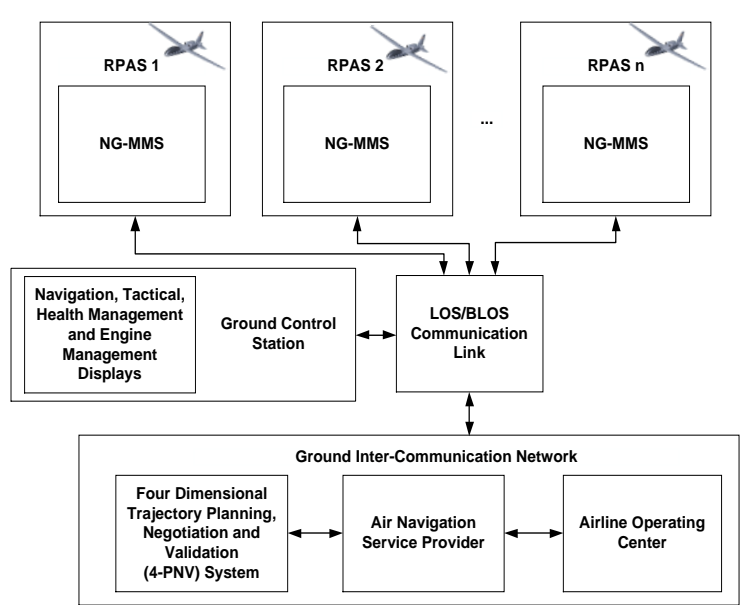

Fig. 5. RPAS CNS+A systems.

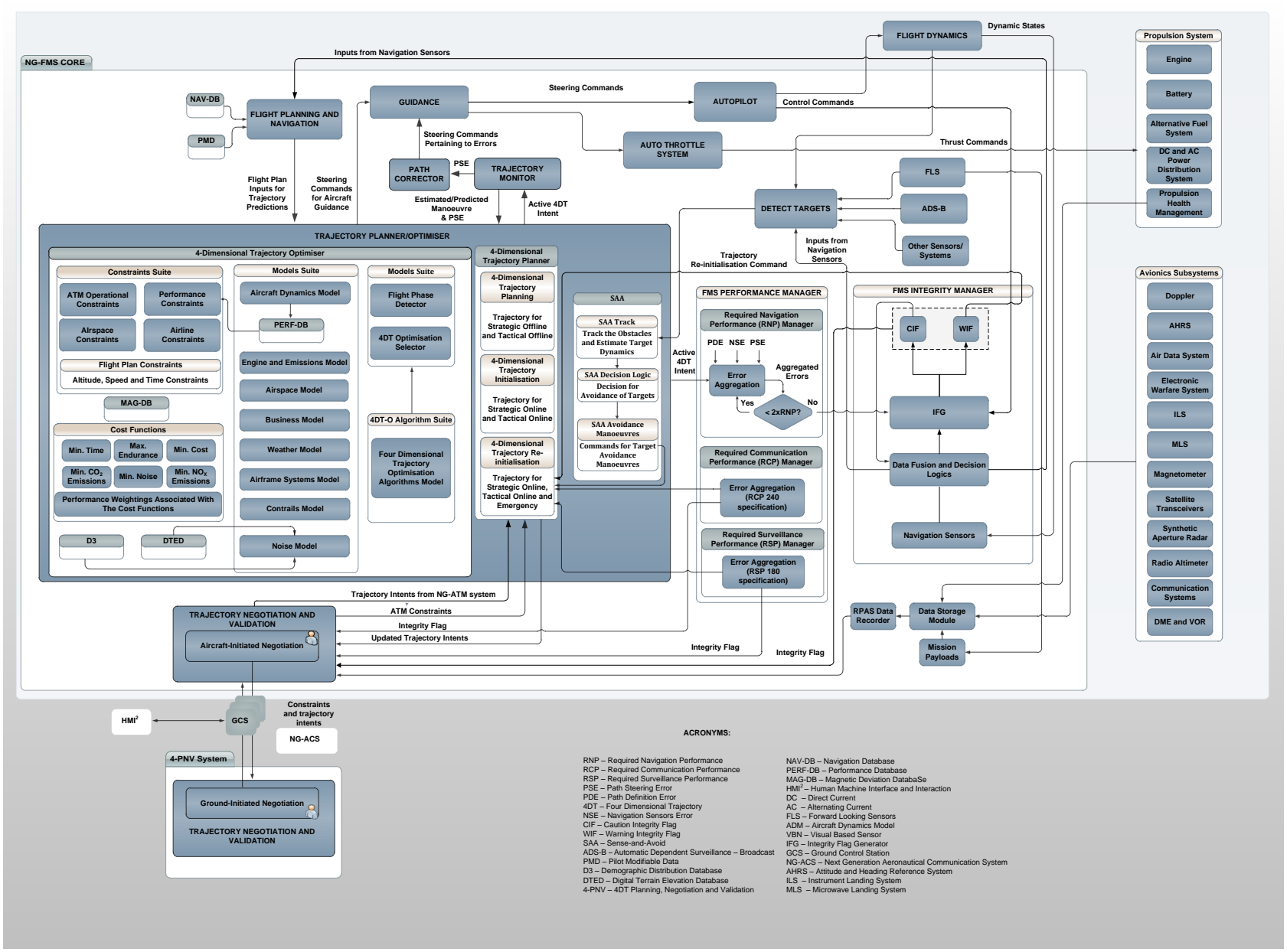

Fig. 6. NG-MMS architecture.

The TDA loop consists of the following functions:

- Track: A group of sensors collect the required data from the environment. Tracking is accomplished by the continuous acquisition of obstacle/intruder data.
- Decision Logics: As the intruder aircraft/obstacle is tracked, suitable decision logics are employed for identifying the possibility of collisions.

- Avoid: Once a possibility of collision is detected, then the on-board computers determine an action to avoid the 
collision by re-generating the 4DT and optimising it against the set constraints and performance parameters.

\section{Multi-ObJective 4DT Optimisation Problem FORMULATION}

From an analytical perspective, the generalised trajectory optimisation problem can be expressed as [10, 11]: "Determine the states $\boldsymbol{x}(t) \in \mathbb{R}^{n}$, the controls $\boldsymbol{u}(t) \in \mathbb{R}^{m}$, the parameters $\boldsymbol{p} \in \mathbb{R}^{q}$, the initial time $t_{0} \in \mathbb{R}$ and the final time $t_{f} \in \mathbb{R} \mid t_{f}>t_{0}$, that optimise the performance indexes:

$$
\boldsymbol{J}=\boldsymbol{\Phi}\left[\boldsymbol{x}\left(t_{0}\right), \boldsymbol{x}\left(t_{f}\right), \boldsymbol{p}\right]+\int_{t_{0}}^{t_{f}} \mathcal{L}[\boldsymbol{x}(t), \boldsymbol{u}(t), \boldsymbol{p}] d t
$$

subject to the dynamic constraints:

$$
\dot{\boldsymbol{x}}(t)=\boldsymbol{f}[\boldsymbol{x}(t), \boldsymbol{u}(t), t, \boldsymbol{p}]
$$

to the path constraints:

$$
\boldsymbol{C}_{\min } \leq \boldsymbol{C}[\boldsymbol{x}(t), \boldsymbol{u}(t), t ; \boldsymbol{p}] \leq \boldsymbol{C}_{\max }
$$

and to the boundary conditions:

$$
\boldsymbol{\Phi}_{\text {min }} \leq \boldsymbol{\Phi}\left[\mathbf{x}\left(\mathrm{t}_{0}\right), \mathbf{x}\left(\mathrm{t}_{f}\right), \mathbf{u}\left(\mathrm{t}_{0}\right), \mathbf{u}\left(\mathrm{t}_{f}\right) ; \mathbf{p}\right] \leq \boldsymbol{\Phi}_{\max }
$$

Various multi-objective optimization strategies were developed and presented in the literature. In particular, multiobjective techniques are globally classified between the ones involving the pre-determination of a unified performance index $J$, as in the a priori approach, and the ones involving the post-processing of the results as in the a posteriori approach [12]. Since we are focussing on real-time optimisation algorithm, the weighted sum method, belonging to the category of a priori articulation of preferences, is adopted. The a priori approach is conceptually represented in Fig. 7.

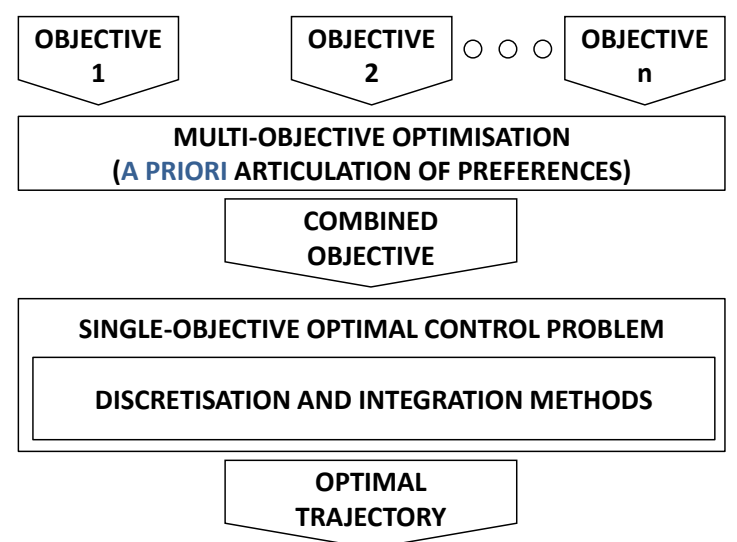

Fig. 7. Block diagram of multi-objective optimisation with a priori articulation of preferences.

Most of the recent studies on trajectory optimization is exploiting the most computationally efficient solvers developed for Optimal Control Problems (OCP), that is, the global orthogonal collocation methods. These methods involve the transcription of the OCP in a Nonlinear Programming (NLP) problem, and are therefore categorized as direct methods. The NLP problem is then solved iteratively by some gradient-based methods, typically associated with relatively quick computational performances, but their nonglobal convergence is an important limitation. The significant number of nonlinearities present in the aircraft flight trajectory models can potentially introduce multiple local minima in the cost functions. A promising solution lays in the adoption of a hybrid approach, with either a pattern search or an evolutionary algorithm performed as a first instance to determine the global convergence region, and subsequently a gradient based iteration to attain local optimality. The current real-time 4DT optimisation algorithm is based on direct global collocation methods, and a hybrid extension is being implemented, by introducing a preliminary discrete pattern search. This last consideration substantially inhibits a pure black-box implementation. Fig. 8 conceptually represents the multimodel multi-objective 4DT optimization algorithm.

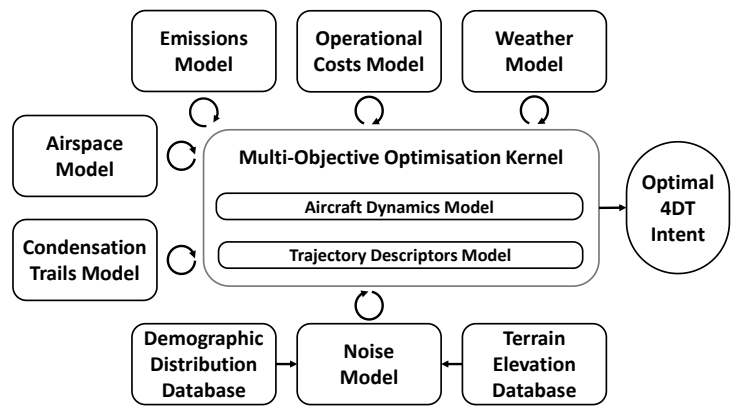

Fig. 8. Conceptual representation of the multi-model multi-objective 4DT optimisation algorithm.

In line with the described OCP formulation, we adopt the following generalised expression of the cost function in Eq. 1 for penalties associated with the transit through a dynamic penalty volume:

$$
J_{i}=\sum_{j=1}^{n_{\text {sect }}} \int\left[P F_{i, j} \cdot\left(\boldsymbol{x}(t) \cap S_{j}(t)\right)\right] d t
$$

where:

$J_{i} \quad=$ Performance/penalty index associated with the $i^{\text {th }}$ objective;

$P F_{i, j}=$ Penalty Factor associated to the $\mathrm{i}^{\text {th }}$ objective in the $\mathrm{j}^{\text {th }}$ penalty volume;

$\boldsymbol{x}(t) \quad=$ Trajectory;

$S_{j}(t)=\mathrm{j}^{\text {th }}$ dynamic penalty volume.

Eq. 5 is used, in the case of 4-PNV and NG-MMS, with suitable forms of the Airspace Model (ASM), Condensation Trails Model (CTM), Weather State Model (WSM) and Noise Emission Model (NEM), by setting the associated penalty factors to adequate values. In our 4-PNV and NGMMS algorithm implementation, the computation of the portion of trajectory inside the penalty volume (Eq. 5) is supported by the Geospatial Data Abstraction Library (GDAL/OGR). The 3-DoF Equations of Motion (EoM) describing the aircraft states and governing the translational movements along the longitudinal, lateral and vertical axes are: 


$$
\begin{aligned}
\frac{d \varphi}{d t} & =\frac{V \cos \gamma \sin \chi+V_{W_{\varphi}}}{R_{M}+h} \\
\frac{d \lambda}{d t} & =\frac{V \cos \gamma \cos \chi+V_{W_{\lambda}}}{\cos \varphi\left(R_{T}+h\right)} \\
\frac{d h}{d t} & =V \sin \gamma+V_{W_{h}} \\
\frac{d V}{d t} & =\frac{\Delta f}{m}-g \sin \gamma \\
\frac{d \gamma}{d t} & =\frac{g(n \cos \phi-\cos \gamma)}{V} \\
\frac{d \chi}{d t} & =\left(\frac{N \cdot g}{V}\right)\left(\frac{\sin \phi}{\cos \gamma}\right) \\
\frac{d m}{d t} & =-c(P, V, h) T(P, V, h) \\
\Delta f= & T(P, V, h)-D(L, V, h)
\end{aligned}
$$

The above Differential Algebraic Equations (DAEs) incorporate three control variables $u=(P, n, \phi)$ where $P$ is the engine power setting, $\mathrm{n}$ is the load factor and $\phi$ is the bank angle. These form the inputs of the dynamic system. The seven aircraft states are $\mathrm{x}=(\mathrm{m}, \varphi, \lambda, \mathrm{h}, \mathrm{V}, \gamma, \chi)$, where $\mathrm{m}$ is the aircraft (variable) mass, $\varphi$ is the geodetic latitude, $\lambda$ is the geodetic longitude, $\mathrm{h}$ is the altitude, $\mathrm{V}$ is the true air speed, $\gamma$ is the flight path angle, $\chi$ is the heading. $R_{M}$ is the meridional radius of curvature, $\mathrm{R}_{\mathrm{T}}$ is the transverse radius of curvature, $\mathrm{W}$ is the wind velocity, $\mathrm{T}$ is the thrust, $\mathrm{D}$ is the drag and $g$ is the nominal acceleration due to gravity of the Earth. Assumptions considered are a rigid body aircraft, nil wing bending effect, rigidly mounted aircraft engine on the vehicle body, zero thrust angle, the location of the aircraft mass in the aircraft centre of gravity, varying mass only as a result of fuel consumption and uniform gravity. Wind effects are considered along the three translational axes of the 3DoF motion equations. The geodetic coordinate reference system used is the World Geodetic System of year 1984 (WGS 84). The lateral path is constructed in terms of segments (straight and turns) and is generally based on the required course change and the aircraft predicted ground speed during the turn. A turn is constructed based on the maximum ground speed of the RPAS during a course change and the turn radius given by:

$$
\mathrm{T}_{\mathrm{R}}=\frac{\mathrm{GS}^{2}}{\mathrm{~g} \times \tan (\phi)}
$$

where GS is the maximum RPAS ground speed during the turn. The turn arc length is given by:

$$
\mathrm{T}_{\mathrm{AL}}=\text { Course }_{\text {change }} \times \mathrm{T}_{\mathrm{R}}
$$

Given the $T_{R}$, the bank angle is calculated from:

$$
\phi=\arctan \left(\frac{\mathrm{GS}^{2}}{\mathrm{~T}_{\mathrm{R}} \times \mathrm{g}}\right)
$$

The NG-MMS computes turn altitude and speed based on the selected altitude by taking into account the predicted wind at that altitude. The bank angle is determined based on RPAS dynamics and airspace configurations. In order to construct the vertical profile, a number of energy balance equations are typically adopted leading to nominal climb/descent, fixed gradients climb/descent, intermediate speed changes and level flight configurations. The integration steps are constrained by the mission profile imposed altitude, speed and time restrictions as well as RPAS performance limitations such as speed and buffet limits, maximum altitude and thrust limits. The data that drives the energy balance equations come from the airframe/ engine dependent thrust, fuel flow, drag and air speed schedule models stored in the PERFormance Data Base (PERFDB). The vertical profile is obtained from the energy method given by:

$$
\frac{d h}{d t}=\frac{(T-D) V_{T}}{G W \times\left(1+\frac{V_{T}}{g} \times \frac{d V_{T}}{d h}\right)}
$$

where $V_{T}$ is the true air speed, GW is the gross weight. The NG-MMS receives the controlled time of arrival target defined by the 4-PNV system, which becomes the Required Time of Arrival (RTA) to be used by the NG-MMS in determining the optimal trajectory states (final time). The Estimated Time of Arrival (ETA) may be assigned to multiple fixes along the flight path. In general, all flight phases allow for incorporating a cost index based on a manually entered CAS / Mach or a combined pair. The cost index allows the mission operators to weigh time, fuel, emissions, noise and other costs. The time cost, $\mathrm{J}_{\text {time }}$ is given by:

$$
\mathrm{J}_{\text {time }}=\mathrm{K}_{\mathrm{t}} \mathrm{t}_{\mathrm{f}}
$$

Fuel consumption optimisation is achieved by minimising the difference between the aircraft initial and final mass, which is included as the $i^{\text {th }}$ DAE of the 3-DoF. Fuel consumption optimisation is achieved by minimising the difference between the aircraft initial and final mass:

$$
\mathrm{J}_{\text {fuel }}=\mathrm{x}_{\mathrm{i}}\left(\mathrm{t}_{\mathrm{f}}\right)-\mathrm{x}_{\mathrm{i}}\left(\mathrm{t}_{0}\right)
$$

In terms of aircraft emissions, although engine design and other factors play an influence on the total amount of emission release, engine emission is generally considered as a function of fuel burn, multiplied by a direct emission factor, @. Hence, the mathematical description of the emission rate, $\mathrm{J}_{\text {emission, }}$ defined with respect to emissions, e and time, $t$ is given by:

$$
\mathrm{J}_{\text {emission }}=\int_{\mathrm{t}_{0}}^{\mathrm{t}_{\mathrm{f}}} \frac{\mathrm{de}}{\mathrm{dt}}=\left(\mathrm{m}\left(\mathrm{t}_{\mathrm{f}}\right)-\mathrm{m}\left(\mathrm{t}_{0}\right)\right) \varrho
$$

The RPAS control loops are illustrated in Figure 9. By considering a typical example of AEROSONDE RPAS, a nonlinear 6-DoF model is adopted as a reference from the AeroSim blockset. In designing a lateral track control strategy for the AEROSONDE RPAS, a viable control strategy is based on the relation: 


$$
\frac{\mathrm{X}_{\text {track }}}{\mathrm{k} \times \mathrm{X}_{\text {track }}}=\frac{\mathrm{Y}_{\text {track }}}{\mathrm{Y}_{\text {track }}}
$$

where $X_{\text {track }}$ and $Y_{\text {track }}$ are the current track position of the RPAS with respect to a TCP. The along-track and crosstrack velocities are obtained from the airspeed and wind speed velocity vectors. The condition to satisfy the control strategy is given by:

$$
\text { Error }=\left(\mathrm{k} \times \mathrm{X}_{\text {track }} \times \mathrm{Y}_{\text {track }}^{\cdot}\right)-\left(\mathrm{Y}_{\text {track }} \times \mathrm{X}_{\text {track }}^{\cdot}\right)
$$

Proportional-Integral-Derivative (PID) and Neuro-Fuzzy logic based controllers are generally implemented for eliminating the error. In order to study the effects of uncertainties on the generated 4DT, a detailed error analysis is performed. The errors might be due to database accuracy degradations, system modelling errors, atmospheric disturbances and subsystem errors. The random errors, which are unpredictable, are quantified to estimate the overall error associated with the position of the aircraft. The system states are modified with the addition of the stochastic term, e(t) and can be represented by:

$$
\dot{x}(t)=f[x(t), u(t), e(t), t]
$$

In order to perform a sensitivity analysis, the sensitivity of a trajectory attribute, $\mathrm{J}$ including consumption of fuel, noise, emissions, etc. is considered with respect to a model parameter, $\Delta$ and is given by:

$$
\Delta=\Delta^{\mathrm{n}}+\mathrm{d} \Delta
$$

The open loop sensitivity of $\mathbf{J}$ is given by:

$$
\frac{\partial \mathrm{J}}{\partial \Delta}_{\mathrm{OL}}=\frac{\mathrm{J}^{\mathrm{OL}}\left(\Delta^{\mathrm{n}}+\mathrm{d} \Delta\right)-\mathrm{J}^{\mathrm{OL}}\left(\Delta^{\mathrm{n}}\right)}{\mathrm{d} \Delta}
$$

The closed loop sensitivity of $\mathrm{J}$ is given by:

$$
\frac{\partial \mathrm{J}}{\partial \Delta}=\frac{\mathrm{J}_{\mathrm{CL}}^{\mathrm{CL}}\left(\Delta^{\mathrm{n}}+\mathrm{d} \Delta\right)-\mathrm{J}^{\mathrm{CL}}\left(\Delta^{\mathrm{n}}\right)}{\mathrm{d} \Delta}
$$

The values of $\mathrm{J}^{\mathrm{OL}}$ and $\mathrm{J}^{\mathrm{CL}}$ are optimised based on the 4DT optimisation algorithm adopted in the MMS. Each performance index provides a quantitative measure of the attainment of a specific objective and different objectives are typically conflicting, and thus the optimisation in terms of two or more objectives typically leads to a number of possible compromise choices, which are still optimal. Therefore, a trade-off is generally introduced in the context of multi-objective trajectory optimisation. In the aviation domain, single and bi-objective optimisation techniques have been exploited for decades but they accounted only for flight time-related costs and fuel-related costs. These techniques have also been implemented in a number of current generation MMS in terms of the Cost Index (CI), which is a scalar value to balance the relative weighting of fuel and time costs. In the NG-MMS, the weightings are varied dynamically among the different flight phases of the flight. While noise criteria can be included in the 4DT optimisation during climb and descent flight phases, optimisation based on emission and fuel cost savings are considered predominantly in the cruise phase. The errors associated with the position of the RPAS are dependent on the error deviations of the ADM parameters given by:

$$
\begin{gathered}
\sigma_{\dot{\mathrm{V}}}=\sqrt{(-\mathrm{g} \cos \gamma)^{2}{\sigma_{\gamma}}^{2}+\left(\frac{\mathrm{Tnorm}}{\mathrm{m}}\right)^{2} \sigma_{\tau}{ }^{2}} \\
\sigma_{\dot{h}}=\sqrt{(\sin \gamma)^{2}{\sigma_{V}}^{2}+{\sigma_{V_{W_{h}}}}^{2}+(V \cos \gamma)^{2}{\sigma_{\gamma}}^{2}}
\end{gathered}
$$

$$
\begin{gathered}
\sigma_{\dot{\gamma}}=\sqrt{\frac{\left[\frac{g(-N \cdot \cos \phi+\cos \gamma)}{V^{2}}\right]^{2} \sigma_{V}^{2}+\cdots}{\left(-\frac{N \cdot g \cdot \sin \phi}{V}\right)^{2} \sigma_{\phi}^{2}+\left(-\frac{g \cdot \sin \gamma}{V^{2}}\right)^{2} \sigma_{\gamma}^{2}}} \\
\sigma_{\dot{\chi}}=\sqrt{\frac{\left(\frac{-N \cdot g \cdot \sin \phi}{\cos \gamma \cdot V^{2}}\right)^{2} \sigma_{V}^{2}+\cdots}{\left(\frac{N \cdot g \cdot \cos \phi}{V \cdot \cos \gamma}\right)^{2} \sigma_{\phi}^{2}+\left(\frac{N \cdot g \cdot \sin \phi \cdot \sin \gamma}{\gamma \cdot \cos \gamma^{2}}\right)^{2} \sigma_{\gamma}^{2}}}
\end{gathered}
$$

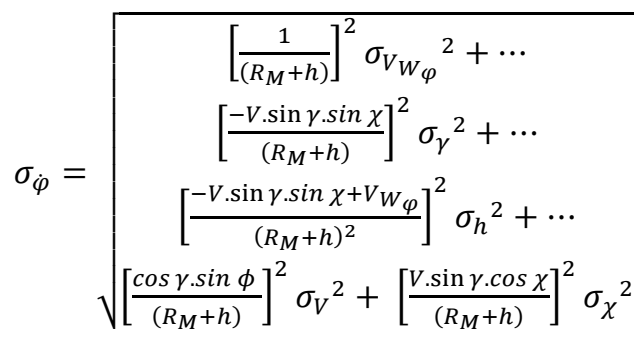

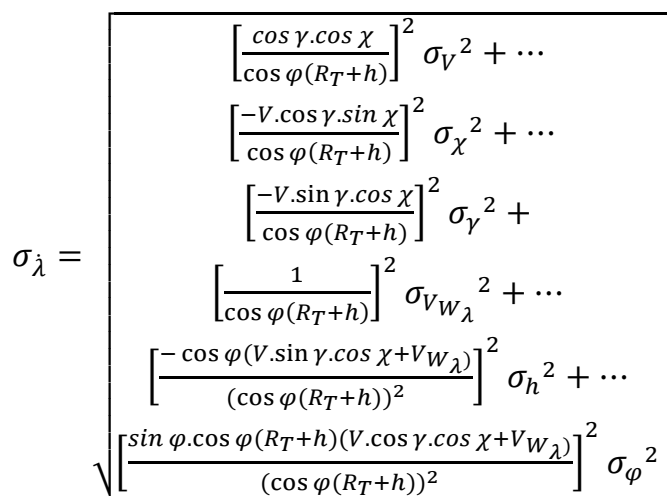

The errors in aircraft positioning are conveniently described by a navigation error ellipsoid. For cooperative and non-cooperative obstacle avoidance and safe-separation maintenance, the overall uncertainty volume is obtained by combining the navigation error ellipsoid with the tracking error ellipsoid and then translating them to unified range and bearing uncertainty descriptors.

\section{Simulation CASE Study}

To illustrate the capability of NG-MMS to generate optimal 4DT, a simulation case study is presented here. An aerial recognition mission involving a loitering phase is simulated. The employed platform is an old variant of the AEROSONDE RPAS. The departure point is St Leonards Airfield (YSLE, S38.166 E144.687), and the working area within which the recognition is performed is the 
AEROSONDE Testing Range, encompassed by Danger Area YMMM/D322A of the Melbourne Flight Information Region (FIR). After the recognition, the RPAS is required to land at the Point Cook Royal Australian Air Force Base (YMPC, S37.933, E144.753). The loitering shall be performed at the test range (S38.210 E144.860), at altitudes between 1000 and $1500 \mathrm{ft}$. Objectives such as fuel consumption, time and emissions, as well as constraints on the path and on the flight envelope are introduced in the trajectory optimization problem based on the information exchanged as a result of the 4DT negotiation process. The climb trajectory is depicted in Fig. 8, while the descent trajectory is depicted in Fig. 9. The operational smoothing algorithm, described in [5] was adopted, and the resulting simplified and flyable trajectory is depicted in magenta. The NG-MMS generated the optimal climb and descent trajectories in 47 seconds. The trajectory corresponding to minimum fuel burn is characterized by a fuel saving of $130 \mathrm{~g}$ when compared to the minimum time case. A stochastic analysis case study was performed to evaluate the potential impact of system uncertainties on the trajectory generation process. The introduction of uncertainties on all nominal parameters, with ranges equal to the standard deviations allows transforming the EoM into stochastic differential equations as given in Eqn. 26 to 31 that are then treated with the Monte Carlo sampling technique and solved using the deterministic optimizer for 100 samples [7]. In these simulations, real weather data obtained with the Global Forecast System (GFS) kindly made available by the National Climatic Data Center (NCDC) of the US National Oceanic and Atmospheric Administration (NOAA) was adopted, as described in [5].

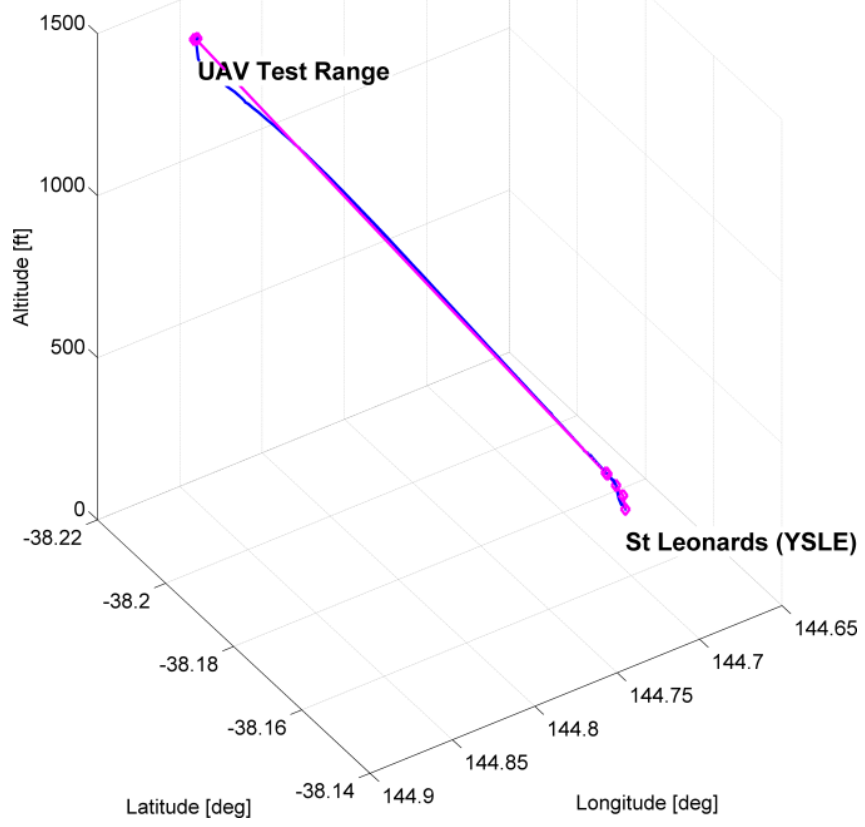

Fig. 9. Results of the 4DT optimisation for climb phase.

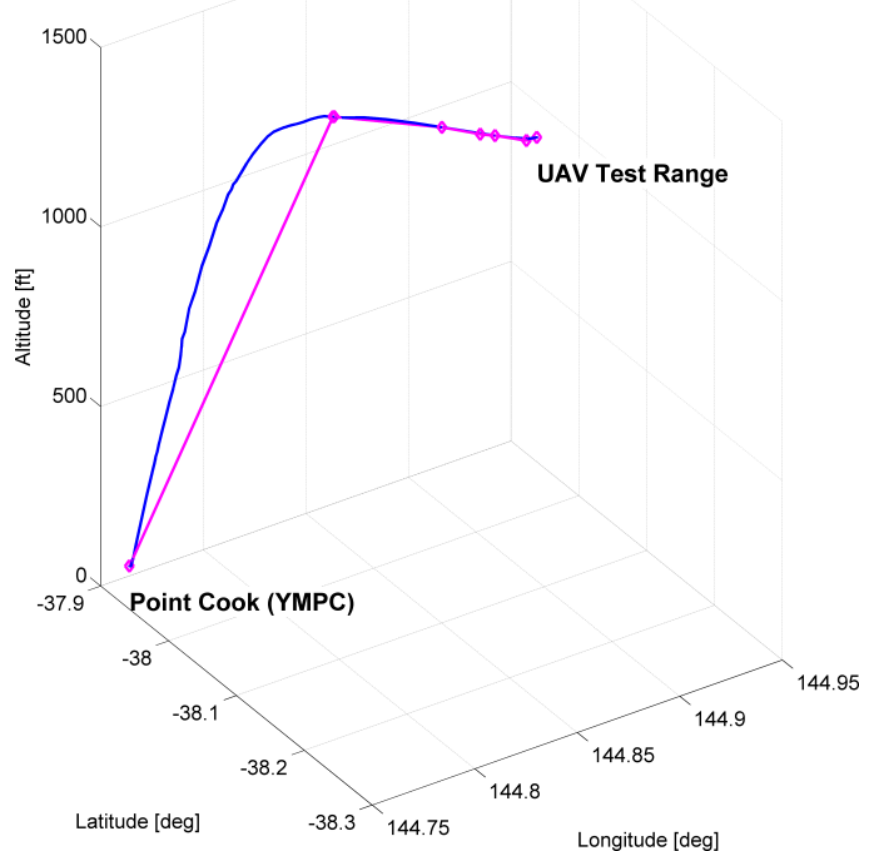

Fig. 10. Results of the 4DT generation for the descent phase.

\section{CONCLUSIONS AND FUTURE WORK}

In this paper we presented the key features of the 4Dimensional Trajectory (4DT) optimisation algorithms implemented in the CNS/ATM and Avionic (CNS+A) systems currently being researched, namely the groundbased 4-Dimensional Trajectory Planning, Negotiation and Validation (4-PNV) system and the airborne Next Generation Mission Management System (NG-MMS) [4, 5]. The overall layout of the multi-objective optimisation algorithm is presented and discussed. A generalised expression for the cost functions associated with penalty volumes is described. This expression is used with suitable forms of the Airspace Model (ASM), Condensation Trails Model (CTM), Weather State Model (WSM) and Noise Emission Model (NEM). Future research will address the detailed derivation of the state equations associated with environmental and economic performance models. Simulation of worst-case scenarios is performed for validation purposes. The proposed 4-PNV/NG-MMS systems are capable to meet the stringent real-time computational requirements for tactical online rerouting operations. Future research will also assess the NG-MMS 4DT intent generation and execution performance in perturbed conditions, based on different navigation architectures and in combination with Avionics-Based Integrity Augmentation strategies [13, 14]. Novel sensing techniques for aviation related pollutants and atmospheric boundary layer/wake turbulence, currently being researched, will provide valuable inputs for dynamic air traffic optimisation.

\section{REFERENCES}

[1] J. E. Robinson III and D. R. Isaacson, "A concurrent sequencing and deconfliction algorithm for terminal 
area air traffic control", in proceedings of $A I A A$ Guidance, Navigation and Control Conference 2000 (GNC 2000), Denver, CO, USA, 2000. DOI: $10.2514 / 6.2000-4473$

[2] H. Erzberger, "The automated airspace concept", in proceedings of 4th USA/Europe Air Traffic Management Research and Development Seminar (ATM2001), Santa Fe, NM, USA, 2001

[3] E. Mueller, "Experimental Evaluation of an Integrated Datalink and Automation-Based Strategic Trajectory Concept", in proceedings of AIAA Aviation Technology, Integration and Operations conference 2007 (ATIO2007), Belfast, Northern Ireland, 2007. DOI: 10.2514/6.2007-7777

[4] S. Ramasamy, R. Sabatini, and A. Gardi, "Novel Flight Management Systems for Improved Safety and Sustainability in the CNS+A Context", in proceedings of Integrated Communication, Navigation and Surveillance Conference (ICNS 2015), Herndon, VA, USA, 2015

[5] A. Gardi, R. Sabatini, T. Kistan, Y. Lim, and S. Ramasamy, "4-Dimensional Trajectory Functionalities for Air Traffic Management Systems", in proceedings of Integrated Communication, Navigation and Surveillance Conference (ICNS 2015), Herndon, VA, USA, 2015

[6] A. Gardi, R. Sabatini, S. Ramasamy, and T. Kistan, "Real-Time Trajectory Optimisation Models for Next Generation Air Traffic Management Systems", Applied Mechanics and Materials, vol. 629, pp. 327-332, 2014. DOI: $10.4028 /$ www.scientific.net/AMM.629.327

[7] S. Ramasamy, R. Sabatini, A. Gardi, and T. Kistan, "Next Generation Flight Management System for RealTime Trajectory Based Operations", Applied Mechanics and Materials, vol. 629, pp. 344-349, 2014. DOI: 10.4028/www.scientific.net/AMM.629.344

[8] A. Gardi, R. Sabatini, S. Ramasamy, and K. de Ridder, "4-Dimensional Trajectory Negotiation and Validation System for the Next Generation Air Traffic Management", in proceedings of AIAA Guidance, Navigation, and Control Conference (GNC 2013), Boston, MA, USA, 2013. DOI: 10.2514/6.2013-4893

[9] D. Sullivan, J. J. Totah, S. S. Wegener, F. Y. Enomoto, C. R. Frost, J. Kaneshige, et al., "Intelligent Mission Management for Uninhabitated Aerial Vehicles", in proceedings of SPIE 5661, Remote Sensing Applications of the Global Positioning System, 2004. DOI: $10.1117 / 12.582446$

[10] A. V. Rao, "Trajectory Optimization", in Encyclopedia of Aerospace Engineering, John Wiley \& Sons Ltd, 2010.

[11] J. T. Betts, Practical methods for optimal control and estimation using nonlinear programming, 2nd ed. vol. 19, SIAM, 2010.

[12] R. T. Marler and J. S. Arora, "Survey of multi-objective optimization methods for engineering", Structural and Multidisciplinary Optimization, vol. 26, pp. 369-395, 2004. DOI: 10.1007/s00158-003-0368-6

[13] R. Sabatini, T. Moore, and C. Hill, "A new avionicsbased GNSS integrity augmentation system: Part 1 Fundamentals", Journal of Navigation, vol. 66, pp. 363384, 2013. DOI: 10.1017/S0373463313000027

[14] R. Sabatini, T. Moore, and C. Hill, "A new avionicsbased GNSS integrity augmentation system: Part 2 Integrity flags", Journal of Navigation, vol. 66, pp. 501522, 2013. DOI: 10.1017/S0373463313000143 